\title{
Moving More and Sitting Less as Healthy Lifestyle Behaviors are Protective Factors for Insomnia, Depression, and Anxiety Among Adolescents During the COVID-19 Pandemic
}

This article was published in the following Dove Press journal:

Psychology Research and Behavior Management

Chunping Lu, ${ }^{\text {, } * ~ X i n l i ~ C h i, ~}{ }^{2, *}$ Kaixin Liang, (iD ${ }^{2}$ Si-Tong Chen, ${ }^{3}$ Liuyue Huang, ${ }^{2}$ Tianyou Guo, ${ }^{2}$ Can Jiao, ${ }^{2}$ Qian $Y u,(1 D)^{4}$ Nicola Veronese, ${ }^{5}$ Fernanda Cunha Soares, ${ }^{6}$ Igor Grabovac, ${ }^{7}$ Albert Yeung, Liye Zou (iD) ${ }^{4}$

'The Greater Bay Area Institute of Educational Research, Shenzhen University, Shenzhen 518060, People's Republic of China; ${ }^{2}$ Center for Lifestyle and Mental Health, Shenzhen University, Shenzhen 518060, People's Republic of China; ${ }^{3}$ Institute for Health and Sport, Victoria University, Melbourne 800I, Australia;

${ }^{4}$ Exercise and Mental Health Laboratory, Shenzhen University, Shenzhen 518060 , People's Republic of China; ${ }^{5}$ Geriatric Unit, Department of Internal Medicine and Geriatrics, University of Palermo, Palermo, Italy; ' ${ }^{6}$ chool of Physical Education,

University of Pernambuco, Recife, PE, Brazil; ${ }^{7}$ Department of Social and Preventive Medicine, Center for Public Health, Medical University of Vienna, Vienna, Wien I090, Austria; ${ }^{8}$ Depression Clinical and Research Program, Massachusetts General Hospital, Boston, MA 02II4, USA

*These authors contributed equally to this work

Correspondence: Liye Zou

Exercise and Mental Health Laboratory,

Shenzhen University, Shenzhen 518060,

People's Republic of China

Tel +86 I88-2343-7684

Email liyezou 123@gmail.com
Purpose: This study aimed to investigate associations of physical activity time (PAT) and sitting time (ST) independently and jointly with insomnia, depressive and anxiety symptoms among Chinese adolescents in the context of COVID-19.

Methods: A cross-sectional study including 965 adolescents (mean age $=15.26, \mathrm{SD}=0.46$ ) was conducted in May 2020. PAT, ST and the symptoms of insomnia, depression and anxiety were assessed with the International Physical Activity Questionnaire Short Form, the Youth Self-Rating Insomnia Scales, the 9-item Patient Health Questionnaire and the Generalized Anxiety Disorder scale via an online survey platform. Logistic regression was used to analyze the association between COVID-19-related fear, PAT, ST and combinations of PAT and ST with insomnia, depressive and anxiety symptoms.

Results: COVID-19-related fear was associated with higher odds of insomnia, depressive and anxiety symptoms. PAT was associated with lower odds of insomnia and depressive symptoms, while ST was associated with higher odds of these three symptoms. After combining PAT and ST, the group with both low PAT and high ST was set as the referent. For insomnia, groups with low ST reported significantly lower odds of symptoms. For depression, groups with high PAT and/or low ST reported lower odds of symptoms. Regarding anxiety, only the group with both high PAT and low ST reported lower odds of symptoms. Generally, group with both high PAT and low ST reported lower odds of the three symptoms than the referent.

Conclusion: COVID-19-related fear was a risk factor for developing insomnia, depressive and anxiety symptoms among Chinese adolescents. Fortunately, moving more and sitting less were good for sleep and mental health in Chinese adolescents during the pandemic. Educators should help adolescents to be more physically active in their daily life in the primary prevention of adolescent insomnia, depression and anxiety.

Keywords: exercise, physical activity, sedentary behavior, sleep, mental health

\section{Introduction}

Since the novel coronavirus disease (COVID-19) swept the world extensively, there have been over 51 million confirmed cases of COVID-19, including more than 1 million deaths globally as of November $13,2020 .{ }^{1}$ As a result, social distancing measures that were implemented to slow the transmission of the COVID-19, together with fear of being infected with the COVID-19, are both linked with mental health problems (MHP) across different age groups including vulnerable 
adolescents. ${ }^{2,3}$ Such problems seem to have gotten worse since the COVID-19 period started, particularly when indirectly comparing to the pre-COVID-19 prevalence because of their unprecedentedly altered lifestyle. ${ }^{4}$ For example, results of a cross-sectional study during part of the COVID-19 epidemic period revealed a high prevalence of insomnia symptoms (21.9\%), depressive symptoms (44.9\%) and anxiety symptoms (38.3\%) among Chinese middle school students ${ }^{5}$ relative to results in previous nonCOVID-19 studies. $^{6-8}$ Of note, insomnia does not only negatively affect daytime function, but also may cause MHP in adolescents. ${ }^{9}$ Additionally, adolescents with MHP tend to be more susceptible to debilitating psychiatric illness in later life. ${ }^{10}$ Given the high prevalence of insomnia, depressive and anxiety symptoms among adolescents during such a stressful life-threatening event (the COVID-19 pandemic), it is essential to assess the prevalence of insomnia, depressive and anxiety symptoms and understand the key modifiable risk/protective factors so that health professionals can timely design effective interventions for the populations at risk of developing these symptoms.

Accumulating evidence has shown that mental health among adolescents can be improved by modifiable lifestyle behaviors, such as physical activity (PA) and sedentary behavior (SB). ${ }^{11,12}$ It is found that physical inactivity was associated with poor mental health during the pandemic. ${ }^{13}$ In contrast, more PA may help decrease the prevalence of depressive and anxiety symptoms. ${ }^{14,15}$ Regular PA during adolescence is not only of great benefit for physical health, ${ }^{16}$ but also has the potential to maintain mental well-being, ${ }^{17}$ while excessive time spent in sitting has been shown to have harmful effects on adolescent mental health. ${ }^{18}$ Thus, promoting PA and limiting SB concurrently might be promising to prevent adolescent mental problems during the COVID-19 and even post-pandemic. ${ }^{19}$ Previous empirical studies have provided the evidence base, for example, in a representative survey of Brazilian adolescents ( $\mathrm{n}=$ 100,839 , mean age $=14.28$ years) where researchers found that less time spent sitting and more time spent physically active was associated with less insomnia. ${ }^{20}$ Such beneficial effects of increased PA and reduced SB were consistently observed in terms of depression and anxiety. ${ }^{21,22}$ While the benefits of PA and problems associated with excessive SB are well documented in the absence of a pandemic, remaining active during the pandemic may be particularly important. ${ }^{17,21}$ Indeed, many researchers have taken the potential role of PA into consideration when it comes to the impact of COVID-19 on MHP. ${ }^{14,23-25}$ For example, a study on Chinese general population found that not exercising was a common risk factor for sleep disorder, depression and anxiety. ${ }^{23}$ Nevertheless, a recent study published in May 2020 found that irregular PA was associated with both higher odds of depression and anxiety and showed some protective effect for adolescent mental health during this global public health emergency. ${ }^{26}$

Despite that valuable information linking PA/SB and mental health before the pandemic, as well as some empirical research conducted during the COVID-19 measuring PA, research about the associations between concurrent PA and SB with mental health among Chinese adolescent during this special period is still limited. Additionally, COVID-19-related fear as a unique variable was observed to associate with increased risks of developing MHP. ${ }^{27,28}$ A recent study showed that fear of COVID-19 causes anxiety, insomnia, and even suicide, reflecting the extreme psychological impacts related to COVID-19. ${ }^{29}$ Although significant associations between fear and the negative mental outcomes have been well documented among adults, ${ }^{48}$ literatures in terms of COVID-19-related fear among adolescents are limited. Thus, such fear perception should be considered as an important factor of symptoms concerned in this study, also an indispensable covariate when examining the relationships between $\mathrm{PA} / \mathrm{SB}$ and MHP in the context of COVID-19. Moreover, researchers have begun to realize that various lifestyle behaviors are codependent and should be investigated simultaneously. ${ }^{30}$ However, most previous studies have separately investigated the relationship(s) of PA or SB with health benefits, especially physical outcome measures. ${ }^{31}$ To fill this void, we therefore aimed to further explore the independent and joint role of PA and/or SB in insomnia, depressive and anxiety symptoms among Chinese adolescents in the context of COVID-19. Collectively, we conducted the present study with three specific aims: (1) to determine the prevalence of insomnia, depressive and anxiety symptoms in a Chinese adolescent sample during the COVID-19; (2) to explore associations between COVID-19-related fear with insomnia, depressive and anxiety symptoms; (3) to investigate associations between $\mathrm{PA} / \mathrm{SB}$ with insomnia, depressive and anxiety symptoms. 


\section{Methods}

\section{Participant Recruitment}

A cross-sectional study on junior middle school students was conducted between May 13 and 20 in 2020, shortly after reopening schools in Yizhou district, Hechi city, Guangxi province, Southern China. Guangxi province is an economically underdeveloped province in southern China, while Hechi city is one of the poorest areas in Guangxi. The rural population accounts for about $77 \%$ in Hechi. ${ }^{32}$ Many studies have found that adolescents living in underdeveloped rural areas are prone to MHP. ${ }^{33,34}$ Hence, it is very important to carry out the psychological survey of middle school students in rural areas (eg, Hechi city) during the COVID-19 pandemic. With a permission from the local educational department (Yizhou district; accounting for $15 \%$ of the population of Hechi city), the survey was conducted on junior middle school students in this district during the pandemic. ${ }^{35}$ There are 30 public middle schools with 38,407 students in Yizhou. ${ }^{35}$ Among all the possible schools in Yizhou, ten schools were randomly selected for our sample recruitment.

Of note, to meet the age requirement of the PA questionnaire and ensure that participants were able to understand the questionnaire content (it is detailed in measurement), only students aged over 15 years in these schools were invited to attend this study, leading to an initial sample size of 1054 students. After removing participants with incomplete and missing data, information of 965 adolescents finally were valid. Based on previous studies on similar topic during the COVID-19 pandemic, the sample was calculated using G*Power 3.1 with the following assumptions: an expected prevalence (40.0\%) of symptoms among Chinese adolescents, 1.5 odds ratio (OR) of symptoms among low PAT or high ST, margin of error of $5 \%$ and power of $80 \%{ }^{36-39}$ This gives a total sample size 778 . The finally recruited sample $(\mathrm{n}=965)$ goes beyond this, which is sufficient for formal analysis (eg, be sufficient to meet the statistical requirements).

\section{Procedure}

With the assistance of the local education authority, official approvals were obtained from the selected schools. In consideration of safety during the pandemic, all participants completed the questionnaires via an online survey platform in which instructions were described. The questionnaires contained scales of PAT, ST, insomnia symptoms, depressive symptoms, anxiety symptoms, and COVID-19-related fear, as well as some socio-demographic information. Prior to data collection, head teachers of the participants have informed participants' guardians regarding the investigation. Subsequently, the informed consent forms were delivered to the participants and their parents. After obtaining their consent, the survey was started. The purpose of this study was explained to all participants and it was highlighted that all the collected data would be analyzed in an aggregated manner with personal information being kept in strict confidentiality. This study was conducted in accordance with the Declaration of Helsinki and approved by both the administration committees of the surveyed schools and the Human Research Ethics Committee of Shenzhen University (Code number: 2,020,005).

\section{Measurements}

\section{Physical Activity and Sitting Time}

Daily PA time and sitting time were self-reported using the International Physical Activity Questionnaire Short Form (IPAQ-SF), which was developed as a surveillance instrument to measure multiple domains of PA. ${ }^{40,41}$ Of note, it has widely been recommended to measure PA of individuals aged between 15 and 65 years. ${ }^{40}$ The IPAQ-SF asks respondents to report the frequency and duration in each PA (vigorous-intensity activities, moderate-intensity activities and walking), as well as the duration of daily sitting during the last 7 days. The Chinese version of the IPAQ-SF has been confirmed adequately reliable and valid in previous studies. ${ }^{41}$

\section{Insomnia Symptoms}

Insomnia symptoms were measured using the Chinese version of the Youth Self-Rating Insomnia Scales (YSIS). ${ }^{42}$ The YSIS consisted of 8 items with each item that is rated on a 5-point scale. A total YSIS score that ranged from 8 to 40 can be obtained, with a higher total score indicating a greater severity over the past month. Participants who scored 22 and above were identified as having insomnia symptoms, whereas participants with score of less than 22 had no insomnia symptoms. The psychometric properties of the YSIS were assessed in a large sample of Chinese adolescents with an internal consistency reliability coefficient of 0.80 and 2-week test-retest reliability coefficient of $0.82 .{ }^{42}$ In the current study, the Cronbach's $\alpha$ coefficient was 0.90 .

\section{Depressive Symptoms}

The Chinese version of the 9-item Patient Health Questionnaire (PHQ-9) was used to measure level of 
depressive symptoms. ${ }^{43}$ Each item that can earn 0 to 3 points $(0=$ "not at all" to $3=$ "nearly every day"), and a total score ranged from 0 to 27 (higher points indicating more severe depressive symptoms). Total scores of 5 , 10,15 , and 20 were identified as the cut-off of mild, moderate, moderately severe and severe symptoms of depression, respectively. Thus, a total score of 5 was set as a cut-off point to categorize participants into two groups: no depressive symptoms (PHQ-9 score $<5$ ) and depressive symptoms (PHQ-9 score $\geq 5$ ). The Chinese version of PHQ-9 has been widely used and well validated in Chinese adolescents, possessing adequate internal consistency of $0.84,{ }^{5,44}$ which is further supported by the current study, with a Cronbach's $\alpha$ coefficient of 0.90 .

\section{Anxiety Symptoms}

Anxiety symptoms were measured using the Chinese version of the Generalized Anxiety Disorder scale (GAD-7), which is an appropriate measure of anxiety symptoms in general. ${ }^{45,46}$ Each item has four response options $(0=$ "Not at all" to 3 = "Nearly every day"). Each participant can obtain a total score that ranged from 0 to 21 , with higher score indicating more severe anxiety. Total scores of greater than or equal to $5,10,15$, and 20 were identified as mild, moderate, moderately severe and severe anxiety, respectively. Thus, with the cut-off point set at 5, participants were categorized into two groups: no anxiety symptoms (GAD-7 score $<5)$ and anxiety symptoms (GAD-7 score $\geq 5$ ). The psychometric properties of the GAD-7 have been validated in Chinese adolescents, ${ }^{5,33,45}$ which is further supported by the current study with a good internal consistency (Cronbach's $\alpha$ coefficient $=0.93$ ).

\section{COVID-19-Related Fear}

Fear was measured using the Chinese version of the Fear of COVID-19 Scale (FCV-19S) ${ }^{47,48}$ It consisted of 7 items, with each item that can be responded on a 5-point Likert scale (strongly disagree $=1$ to strongly agree $=5$ ). Its total score (summation of individual response items) ranged from 7 to 35 , with higher scores indicating greater COVID-19-related fear. The psychometric properties of the FCV-19S have been confirmed in a Chinese population with a good internal consistency reliability $(\alpha=0.92)$, composite reliability $(\mathrm{CR}=0.92)$ and good structural validity. ${ }^{47}$ Good internal consistency was also presented in this study with a Cronbach's $\alpha$ coefficient of 0.94 .

\section{Socio-Demographic Information}

Socio-demographic information was collected via a selfreported questionnaire, including age, gender (male/ female). Their family economic status was also collected, indicated by household monthly income per person (Chinese currency [Yuan as unit] - RMB: $<2000 / 2000$ $3000 / \geq 3000)$ in this study. Additionally, they reported their left-behind status (left-behind/non-left-behind; left-behind children are prevalent in modern China, referring to the children who are under 18 years old and left at rural residences for at least 6 months by either one or both of parents migrating to work in cities). Studies have indicated high risk of mental problems among Chinese left-behind children. $^{34,49}$

\section{Statistical Analyses}

Descriptive analyses were performed for participant characteristics. Descriptive data are presented as mean with standard deviation (SD) for continuous variables and frequency with percentage for categorical variables.

Here, according to the median values in this sample, participants were categorized based on the cut-off points of $1.5 \mathrm{~h} / \mathrm{d}$ for PAT and $4 \mathrm{~h} / \mathrm{d}$ for ST, respectively. Thus, the participants were divided into two categories for PAT (low PAT or high PAT). Similarly, the participants were classified into two categories for ST (low ST or high ST). Then, the participants were cross-tabulated into $4(2 \times 2)$ groups (ie, Group I : low PAT + high ST; Group II : low PAT + low ST; Group III: high PAT + high ST; Group IV: high PAT + low ST).

Logistic regressions were used to examine the associations between COVID-19-related fear, PAT, ST and combination of PAT and ST with the symptoms of insomnia, depression and anxiety. Socio-demographic factors (ie, age, gender, left-behind status, and household monthly income per person) and COVID-19-related fear were included as confounding variables when examining the independent and joint associations between PAT and ST with these symptoms. To examine the independent associations between PA/SB with the symptoms of insomnia, depression and anxiety, we also mutually adjusted exposures (PAT and ST) for each other (ie, when PAT was modeled as the main exposure, the analysis was adjusted for ST, and when ST was modeled as the main exposure, the analysis was adjusted for PAT). Next, the joint associations between PAT and ST with the symptoms of insomnia, depression and anxiety were examined. Using Group I (low PAT + high ST) as the referent, 
we compared the odds ratios (OR) of symptoms between Group I and other groups after adjusting for controlled variables. All analyses were performed using SPSS for Windows, version 26.0. The statistical significance was set at $p<0.05$ (two-tailed) for interpretation of the results.

\section{Results}

The final sample in this study consisted of 965 adolescents aged 15-17 years (mean age: $15.26 \pm 0.46$ ), of which females accounted for $42.4 \%$. Of all participants, $49.9 \%$ of participants were categorized to low PAT while 54.0\% of participants were recognized as high ST. Meanwhile, $34.9 \%, 45.7 \%$ and $34.4 \%$ participants reported insomnia, depressive and anxiety symptoms in this study,

Table I Descriptive Characteristics of the Participants

\begin{tabular}{|c|c|}
\hline Variables & $\begin{array}{l}\text { n (\%) or Mean } \pm \\
\text { SD }\end{array}$ \\
\hline Age & $15.26 \pm 0.46$ \\
\hline \multicolumn{2}{|l|}{ Gender } \\
\hline Male & $556(57.6)$ \\
\hline Female & $409(42.4)$ \\
\hline \multicolumn{2}{|l|}{ Left-behind status } \\
\hline Left-behind & $305(31.6)$ \\
\hline Non-left-behind & $660(68.4)$ \\
\hline \multicolumn{2}{|c|}{$\begin{array}{l}\text { Household monthly income per person } \\
\text { (yuan) }\end{array}$} \\
\hline$<2000$ & $334(34.6)$ \\
\hline 2000-3999 & $416(43.1)$ \\
\hline$\geq 4000$ & $215(22.3)$ \\
\hline COVID-19-related fear & $|7.4| \pm 7.29$ \\
\hline \multicolumn{2}{|l|}{ Physical activity time (PAT) (h/d) } \\
\hline$<1.5 \mathrm{~h} / \mathrm{d}$ (low PAT) & $482(49.9)$ \\
\hline$\geq 1.5 \mathrm{~h} / \mathrm{d}$ (high PAT) & $483(50.1)$ \\
\hline \multicolumn{2}{|l|}{ Sitting time $(\mathbf{S T})(\mathrm{h} / \mathrm{d})$} \\
\hline$<4 \mathrm{~h} / \mathrm{d}$ (low ST) & $444(46.0)$ \\
\hline$\geq 4 \mathrm{~h} / \mathrm{d}$ (high ST) & $521(54.0)$ \\
\hline \multicolumn{2}{|l|}{ Insomnia symptoms } \\
\hline With insomnia symptoms & $337(34.9)$ \\
\hline Without insomnia symptoms & $628(65.1)$ \\
\hline \multicolumn{2}{|l|}{ Depression symptoms } \\
\hline With depressive symptoms & $44 \mid(45.7)$ \\
\hline Without depressive symptoms & $524(54.3)$ \\
\hline \multicolumn{2}{|l|}{ Anxiety symptoms } \\
\hline With anxiety symptoms & $332(34.4)$ \\
\hline Without anxiety symptoms & $633(65.6)$ \\
\hline
\end{tabular}

respectively. More detailed descriptive characteristics of the participants are shown in Table 1.

Table 2 presents the independent associations between COVID-19-related fear, PAT and ST with insomnia, depressive and anxiety symptoms. Higher COVID-19-related fear was significantly related with the occurrence of insomnia symptoms $(\mathrm{OR}=1.03$ [1.01-1.05], $p<0.01)$, depressive symptoms $(\mathrm{OR}=1.03$ [1.01-1.05], $p=0.001)$ and anxiety symptoms $(\mathrm{OR}$ $=1.06$ [1.04-1.08], $p<0.001)$. Regarding the independent associations of PAT and ST with insomnia, depressive and anxiety symptoms, participants with high PAT reported less opportunities to develop insomnia symptoms $(\mathrm{OR}=0.71[0$.$54-0.95], p<0.05)$ and depressive symptoms $(\mathrm{OR}=0.71$ [0.54-0.93], $p<0.05)$ than those with low PAT after adjusting for age, gender, left-behind status, household monthly income per person, COVID-19-related fear and ST. In contrast, participants with high ST were more likely to develop insomnia symptoms $(\mathrm{OR}=1.79$ [1.36-2.37], $p<0.001)$, depressive symptoms $(\mathrm{OR}=1.65[1.26-2.16], p<0.001)$ and anxiety symptoms $(\mathrm{OR}=1.47[1.11-1.94], p<0.01)$ than those with low ST after adjusting for age, gender, left-behind status, household monthly income per person, COVID-19-related fear and PAT.

Table 3 shows the joint associations of PA and SB with the symptoms of insomnia, depression and anxiety. For insomnia, Group II $(\mathrm{OR}=0.56$ [0.38-0.83], $p<0.01)$ and Group IV $(\mathrm{OR}=0.40$ [0.27-0.59], $p<0.001)$, namely the two groups with low SB, reported significantly lower odds than the reference group (ie, Group I ). For depression, Group II $(\mathrm{OR}=0.55$ [0.38-0.80], $p<0.01)$, Group III $(\mathrm{OR}=0.65[0.45-0.93], p<0.05)$ and Group IV $(\mathrm{OR}=$ 0.43 [0.30-0.63], $p<0.001)$, namely the groups with high PA and/or low SB, were all significantly associated with lower odds of symptoms than the reference group. For anxiety, only the group with high PAT and low ST concurrently (ie, Group IV) was associated with lower odds of symptoms than reference group $(\mathrm{OR}=0.65$ [0.43-0.97], $p<0.05)$. Generally, compared to the reference group with both unhealthy behaviors (ie, low PAT + high ST), participants with both healthy behaviors (ie, high PAT + low ST) demonstrated significantly lower prevalence of insomnia symptoms $(\mathrm{OR}=0.40$ [0.27-0.59], $p<0.001)$, depressive symptoms $(\mathrm{OR}=0.43[0.30-0.63], p<0.001)$ and anxiety symptoms $(\mathrm{OR}=0.65$ [0.43-0.97], $p<0.05)$.

\section{Discussion}

Using a sample of Chinese adolescents during the COVID-19, this study aimed to investigate the 
Table 2 Independent Associations of Physical Activity Time (PAT) and Sitting Time (ST) with the Odds Ratios of Insomnia, Depressive and Anxiety Symptoms

\begin{tabular}{|c|c|c|c|c|}
\hline & & $\begin{array}{l}\text { Insomnia } \\
\text { Symptoms }\end{array}$ & $\begin{array}{l}\text { Depressive } \\
\text { Symptoms }\end{array}$ & $\begin{array}{l}\text { Anxiety } \\
\text { Symptoms }\end{array}$ \\
\hline Age & & $1.30(0.97-1.75)$ & $0.97(0.72-1.29)$ & $1.25(0.93-1.69)$ \\
\hline Gender & $\begin{array}{l}\text { Male } \\
\text { Female }\end{array}$ & $\begin{array}{l}\text { I (ref) } \\
1.37(1.03-1.82)^{*}\end{array}$ & $\begin{array}{l}\text { I (ref) } \\
\text { I.66 (1.26-2.19)*** }\end{array}$ & $\begin{array}{l}\text { I (ref) } \\
1.60(1.20-2.13)^{* *}\end{array}$ \\
\hline Left-behind status & $\begin{array}{l}\text { Non-Left-behind } \\
\text { Left-behind }\end{array}$ & $\begin{array}{l}\text { I (ref) } \\
1.27(1.03-1.46)\end{array}$ & $\begin{array}{l}\text { I (ref) } \\
\text { I.43 (I.24-I.57)*** }\end{array}$ & $\begin{array}{l}\text { I (ref) } \\
\text { I.32 (I.09-1.49)* }\end{array}$ \\
\hline Household monthly income per person (yuan) & $\begin{array}{l}<2000 \\
2000-3999 \\
\geq 4000\end{array}$ & $\begin{array}{l}\text { I (ref) } \\
0.99(0.72-1.35) \\
0.77(0.53-1.13)\end{array}$ & $\begin{array}{l}\text { I (ref) } \\
0.93(0.68-1.25) \\
0.82(0.57-1.18)\end{array}$ & $\begin{array}{l}\text { I (ref) } \\
0.87(0.63-1.18) \\
0.75(0.51-1.10)\end{array}$ \\
\hline \multicolumn{2}{|l|}{ COVID-19-related fear } & $1.03(1.01-1.05)^{* *}$ & $1.03(1.01-1.05)^{* *}$ & $1.06(1.04-1.08)^{* * *}$ \\
\hline PA & $\begin{array}{l}\text { Low PAT } \\
\text { High PAT }\end{array}$ & $\begin{array}{l}\text { I (ref) } \\
0.7 \mid(0.54-0.95)^{*}\end{array}$ & $\begin{array}{l}\text { I (ref) } \\
0.7 \mid(0.54-0.93)^{*}\end{array}$ & $\begin{array}{l}\text { I (ref) } \\
0.97(0.73-1.29)\end{array}$ \\
\hline Sitting & $\begin{array}{l}\text { Low ST } \\
\text { High ST }\end{array}$ & $\begin{array}{l}\text { I (ref) } \\
\text { I.80 }(0.54-0.95)^{* * *}\end{array}$ & $\begin{array}{l}\text { I (ref) } \\
\text { I.65 (1.26-2.16)*** }\end{array}$ & $\begin{array}{l}\text { I (ref) } \\
\text { I.47 (I.II-I.94)** }\end{array}$ \\
\hline
\end{tabular}

Notes: Data are presented as OR $(95 \% \mathrm{Cl}) .{ }^{*} p<0.05,{ }^{*} p<<0.01$, ${ }^{* * *} p<0.00$ I. PAT or ST was mutually adjusted according to the independent variable (ie, when PAT was modeled as the main independent variable, the analysis was adjusted for ST, and when ST was modeled as the main independent variable, the analysis was adjusted for PAT).

Table 3 Joint Associations of Physical Activity (PAT) and Sitting Time (ST) with the Odds Ratios of Insomnia, Depressive and Anxiety Symptoms

\begin{tabular}{|l|l|l|l|}
\hline & Insomnia Symptoms & Depressive Symptoms & Anxiety Symptoms \\
\hline Group I & I (ref) & I (ref) & I (ref) \\
\hline Group II & $0.56(0.38-0.83)^{* *}$ & $0.55(0.38-0.80)^{* *}$ & $0.73(0.50-I .10)$ \\
\hline Group III & $0.72(0.50-1.03)$ & $0.65(0.45-0.93)^{*}$ & $1.05(0.72-1.52)$ \\
\hline Group IV & $0.40(0.27-0.59)^{* * *}$ & $0.43(0.30-0.63)^{* * *}$ & $0.65(0.43-0.97)^{*}$ \\
\hline
\end{tabular}

Notes: Data are presented as OR $(95 \% \mathrm{Cl}) .{ }^{*} p<0.05, * * p<0.0$ I, ***p $<0.001$. Group I : low PAT + high ST; Group II : low PAT + low ST; Grouplll: high PAT + high ST; Group IV: high PAT + low ST. Using Group I as the referent, the odds ratios of the three symptoms between Group I and other groups were compared after adjusting for age, gender, household monthly income per person, left-behind status, COVID-19-related fear.

prevalence of insomnia, depressive and anxiety symptoms, independent and joint associations between PAT and ST with these symptoms, as well as the associations between COVID-19-related fear with these symptoms. The prevalence of insomnia, depressive and anxiety symptoms in this sample was $34.9 \%, 45.7 \%$ and $34.4 \%$, respectively. More COVID-19-related fear was correlated with higher odds of insomnia, depressive and anxiety symptoms, while high PAT and low ST were associated with lower odds of insomnia and depressive symptoms. Compared to the reference group with unhealthy behaviors (low PAT and high ST), the group with both healthy lifestyle behaviors (high PAT and low ST) was significantly associated with lower odds of insomnia, depressive and anxiety symptoms. Additionally, only the group with concurrent high PAT and low ST presented significantly lower odds than the reference group. Possible explanations for these results are presented in detail below.

Prevalence of insomnia, depressive and anxiety symptoms in this study was generally high, which reflects the psychological impact of the pandemic to some extent. One reason for such a high prevalence may be because all the participants were junior high school students over 15 years old; thus, a large proportion of our participants were students in Grade 3, who were facing the pressure of senior middle school entrance examination (the most important examination for junior middle school students). Besides, 
our sampling process was conducted in Guangxi province, one of the economically backward areas in China, nearly a third of the students were left-behind children. Previous research has found that left-behind children were more likely to develop mental health problems as they experience separation from harmonious parent-child interactions. $^{49}$ A relatively low cut-off point of 5 of the PHQ-9 and GAD-7 may also explain the high prevalence. Nevertheless, our results were similar to a large-scale study with 11,835 Chinese adolescents conducted in March 2020 (two months earlier than this study), which assessed depressive and anxiety symptoms by the PHQ-9 and GAD-7 and adopted the same cut-off point as our study. This is a warning that negative impact of the pandemic on adolescent mental health, may be continuous and long term despite that COVID-19 situation has been better in China.

Results in the current study indicated that greater COVID-19-related fear was significantly associated with higher levels of insomnia, depressive and anxiety symptoms. This is consistent with a pre-COVID-19 study indicating that people who suffered from fear tended to develop insomnia symptoms. ${ }^{50}$ Recent studies conducted during the COVID-19 also suggested that fear of COVID19 was associated with psychological distress and life dissatisfaction. $^{28}$ Aggregated fear of COVID-19 may even lead to suicide. ${ }^{29}$ Taken together, fear caused by lifethreatening event like COVID-19- would increase the probability of developing mental health problems, indirectly reflecting the psychological impact of the COVID19. The fear and psychological distress related to the pandemic has been found to be associated with the stressors such as frustration, inadequate information, and problematical media exposure. ${ }^{51}$ Thus, parents and educators should pay attention to adolescents' fear to COVID-19 and their consumption of pandemic-related media coverage, and help adolescents with proper knowledge on the COVID-19.

Since the pandemic has been such a great threat to the mental health of adolescents, it is very important to figure out the protective factors to prevent or intervene the potential MHP. Generally, it is considered that either high PA or low SB is healthy for adolescents. ${ }^{52-54}$ This study conducted during the COVID-19 also supported this idea. Specifically, participants with high PAT were less likely to develop insomnia and depressive symptoms than counterparts with low PAT, while high ST group was more likely to develop insomnia, depressive and anxiety than those with low ST even after mutually adjusting for each other of PAT and ST. Although our data cannot render a causal inference about the association, previous research can provide some indications of a potentially causal relationship, which would underpin our assertions. In terms of PA, for example, a randomized controlled trial indicated that 30-minute morning running during weekdays for three consecutive weeks had positive impact on sleep quality and psychological functioning among healthy adolescents relative to a control group. ${ }^{55}$ In addition, a prospective cohort study found that increasing ST (measured by anti-graph) was associated with greater level of depression at age 18 years relative to its baseline assessment of children aged 12-16 years. ${ }^{12}$ Besides, it was reported that a one-week sedentary behavior-inducing intervention had deleterious effects on anxiety among an active youth population as compared with a control group. ${ }^{56}$

Despite extensive research on the efficacy of PA/SB, the potential mechanism through which they produce mental health benefits among adolescents remains unclear. Nevertheless, biological, psychological and behavioral mechanisms have been suggested for the relationships between PA with mental health of young people. ${ }^{57}$ Biological mechanism hypothesis suggests that PA improves mental health via structural and functional changes in the brain, such as improving levels of moodregulating neurotransmitters in the brain or the production of cortisol. ${ }^{58-60} \mathrm{PA}$ was also found to be a mediator in the relationship between inflammation and depression in adults, but relevant evidence from adolescents is limited. ${ }^{61,62}$ Psychological mechanism hypothesis suggests that PA makes it more accessible for social interaction and social support, improving the self-esteem, self-efficacy and perceived competence and improvements in body image. $^{63,64}$ Behavioral mechanism hypothesis suggested that PA may improve self-regulation and coping skills which may help effectively stay positive mentally. ${ }^{57}$ Regarding the positive impact on sleep, it is expected that by energy consumption, PA would increase the need for sleep at night to reduce metabolic requirements for energy conservation and body restoration. ${ }^{65}$ Moreover, physically active individuals tend to maintain other healthy behaviors, such as favorable diet patterns which are associated with better sleep quality and mental health. ${ }^{66,67}$ Regarding the undesirable influence of SB, previous research has suggested that it is also associated with inflammation, which is a core feature of major depression. ${ }^{68,69}$ A meta-analysis suggested that high levels 
of SB are associated with higher levels of anxiety symptoms, and the associations remain largely similar, regardless of age, health status and measurements. ${ }^{70}$ There is some provisional evidence to suggest that standing or breaking up prolonged periods of sitting might help alleviate the harmful effect of SB on metabolism in adolescents, ${ }^{71}$ but more research to clarify the mechanism of SB on sleep and mental health are needed.

Taken together, owing to the independent impact of increased PA or limited SB, it is expected that increasing PA and limiting SB concurrently would be a better protective factor to improve sleep and mental health than only focusing on PA or SB among adolescents. This expectation is supported by the main findings of the joint roles of PA and SB in this study: combinations with healthy behaviors (high PAT + low ST) were all significantly associated with lower odds of insomnia, depression and anxiety symptoms than the combinations with unhealthy behaviors (low PAT + high $\mathrm{SB}$ ). The presence of health-promoting behaviors may produce a cumulative effect on mental health outcomes. ${ }^{31}$ Most current research findings have suggested that meeting behavior recommendations (eg, adolescents aged 14-17 years need to engage in at least 60 min per day of moderate-to-vigorous PA) may aid in attaining better physical and mental health in adolescents. ${ }^{30,72}$ In fact, as mentioned before, many researchers have expressed their interest in modifiable lifestyle behaviors when the psychologists seek to figure out ways to combat the burden on mental health caused by the pandemic. ${ }^{26,73}$ Therefore, in the follow-up work, more research should look into the related factors with the time distribution on PA and SB among adolescents as the pandemic is still going on.

Another interesting finding in this study was found in the association between PA and anxiety symptoms: no significant difference on anxiety existed between high PAT and low PAT. This may implicate the weak association between PA and anxiety, which is consistent with a conclusion given in a review paper that PA only has a small beneficial effect for alleviating anxiety symptoms. ${ }^{74}$ Moreover, after combining PA and SB, as compared to the reference group (ie, low PAT + high ST), only the group with high PAT and low ST concurrently was associated with lower odds of anxiety symptoms, while only the groups with low ST were associated with lower odds of insomnia symptoms. Our results suggest that a high level of PA may not alleviate the effect of excessive SB on mental health outcomes. Findings in the present study partially agrees with a previous study, which found that adolescents with concurrent high PA and high SB might be also experiencing poor mental health. ${ }^{75}$ Besides, a cross-sectional study demonstrated that screen time, closely related with sitting time, had greater impact on mental health than PA. ${ }^{76}$ However, compared with the attention paid to PA, information on the relationships between SB and mental health is less available, which highlights the importance to investigate the underlying mechanism of SB on mental health in future research. More importantly, these findings reaffirm that when non-health-promoting behaviors coexist with healthpromoting behaviors, the benefit of healthy behaviors may be eliminated. ${ }^{31}$ Thus, future interventions should consider how behaviors cluster and interact with each other. Rather than putting too much emphasis on one single lifestyle behavior, especially in the context of public health emergency, our findings reinforce the importance of promoting PA and limiting SB concurrently in preventing symptoms of insomnia, depression and anxiety in Chinese adolescents during the COVID-19 pandemic.

Some limitations in our study should be mentioned. First, the study involved only junior middle school students in Yizhou district, Hechi city. Even though we have included left-behind status and household income as confounding factors, one should be cautious in generalizing our findings to all Chinese adolescents. Future studies are encouraged to replicate or negate our research findings based on young population with a wider range of ages and socioeconomic status. Second, the nature of cross-sectional design allows no inferences on the causality of any associated factor. More comprehensive longitudinal studies are needed to better understand the complex and potentially reciprocal relationship between the variables (ie, PA, SB, insomnia, depressive and anxiety symptoms). Third, the self-reported information may cause bias due to the social desirability effect and memory error. Studies with larger sample size and objective measure are highly recommended in future studies. ${ }^{77}$ Fourth, although the presence of mental problems was assessed by the standardized questionnaires, these measures are not completely equivalent to clinical diagnoses; thus, future studies with diagnostic interviews should be used.

\section{Conclusion}

The present study found that COVID-19-related fear is significantly associated with the prevalence of insomnia, depressive and anxiety symptoms. Besides, it provided further evidence that high PA and low SB contributed to better sleep and related mental outcomes, while the co-existence of these two behaviors could confer a greater benefit in adolescents. This study suggested that moving more and sitting less is good for sleep quality and mental health for Chinese adolescents 
during COVID-19 pandemic. For the prevention and intervention of insomnia, depression and anxiety, it is worthwhile to pay attention to the joint roles of PA promotion and SB reduction in adolescents, by encouraging adolescents to be physically active and decrease sedentary behavior. Future studies with longitudinal design and objective measures are encouraged to provide more information on this topic.

\section{Acknowledgments}

The authors are extremely grateful to all the participants who took part in this study.

\section{Disclosure}

The authors report no conflicts of interest in this work.

\section{References}

1. World Health Organization. WHO coronavirus disease (COVID-19) dashboard. 2020

2. Brooks SK, Webster RK, Smith LE, et al. The psychological impact of quarantine and how to reduce it: rapid review of the evidence. Lancet. 2020;395(10227):912-920. doi:10.1016/S0140-6736(20)30460-8

3. Ornell F, Schuch JB, Sordi AO, Kessler FHP. Pandemic fear and COVID-19: mental health burden and strategies. Braz J Psychiatry. 2020;3(42).

4. Guessoum SB, Lachal J, Radjack R, et al. Adolescent psychiatric disorders during the COVID-19 pandemic and lockdown. Psychiatr Res. 2020;291:113264. doi:10.1016/j.psychres.2020.113264

5. Zhou S, Wang L, Yang R, et al. Sleep problems among Chinese adolescents and young adults during the coronavirus-2019 pandemic. Sleep Med. 2020. doi:10.1016/j.sleep.2020.06.001

6. Tang X, Tang S, Ren Z, Wong DFK. Prevalence of depressive symptoms among adolescents in secondary school in mainland China: a systematic review and meta-analysis. $J$ Affect Disorders. 2019;245:498-507. doi:10.1016/j.jad.2018.11.043

7. Gong Q, Li S, Wang S, Li H, Han L. Sleep and suicidality in school-aged adolescents: a prospective study with 2-year follow-up. Psychiatry Res. 2020;287:112918. doi:10.1016/j.psychres.2020.112918

8. Xiong W, Liu H, Gong P, et al. Relationships of coping styles and sleep quality with anxiety symptoms among Chinese adolescents: a cross-sectional study. $J$ Affect Disord. 2019;257:108-115. doi:10.1016/j.jad.2019.07.032

9. Chan NY, Zhang J, Tsang CC, et al. The associations of insomnia symptoms and chronotype with daytime sleepiness, mood symptoms and suicide risk in adolescents. Sleep Med. 2020;74:124-131. doi:10.1016/j.sleep.2020.05.035

10. Ssegonja R, Alaie I, Philipson A, et al. Depressive disorders in adolescence, recurrence in early adulthood, and healthcare usage in mid-adulthood: a longitudinal cost-of-illness study. J Affect Disord. 2019;258:33-41. doi:10.1016/j.jad.2019.07.077

11. Ayala AMC, Salmon J, Dunstan DW, Arundell L, Timperio A. Does light-intensity physical activity moderate the relationship between sitting time and adiposity markers in adolescents? $J$ Sport Health Sci. 2020.

12. Kandola A, Lewis G, Osborn D, Stubbs B, Hayes JF. Depressive symptoms and objectively measured physical activity and sedentary behaviour throughout adolescence: a prospective cohort study. Lancet Psychiatr. 2020;7(3):262-271. doi:10.1016/S2215-0366(20)30034-1
13. Werneck AO, Silva DR, Malta DC, et al. Changes in the clustering of unhealthy movement behaviors during the COVID-19 quarantine and the association with mental health indicators among Brazilian adults. Transl Behav Med. 2020. doi:10.1093/tbm/ibaa095

14. Fullana MA, Hidalgo-Mazzei D, Vieta E, Radua J. Coping behaviors associated with decreased anxiety and depressive symptoms during the COVID-19 pandemic and lockdown. $J$ Affect Disord. 2020;275:80-81. doi:10.1016/j.jad.2020.06.027

15. Nguyen HC, Nguyen MH, Do BN, et al. People with suspected covid-19 symptoms were more likely depressed and had lower health-related quality of life: the potential benefit of health literacy. J Clin Med. 2020;9(4):965. doi:10.3390/jcm9040965

16. de Oliveira RGA, Guede DP, Rosenfeld CS. Physical activity, sedentary behavior, cardiorespiratory fitness and metabolic syndrome in adolescents: systematic review and meta-analysis of observational evidence. PLoS One. 2016;11(12):e0168503. doi:10.1371/journal. pone. 0168503

17. Biddle SJH, Ciaccioni S, Thomas G, Vergeer I. Physical activity and mental health in children and adolescents: an updated review of reviews and an analysis of causality. Psychol Sport Exerc. 2019;42:146-155. doi:10.1016/j.psychsport.2018.08.011

18. Suchert V, Hanewinkel R, Isensee B. Sedentary behavior, depressed affect, and indicators of mental well-being in adolescence: does the screen only matter for girls? J Adolesc. 2015;42:50-58. doi:10.1016/ j.adolescence.2015.03.014

19. Ellul MA, Benjamin L, Singh B, et al. Neurological associations of COVID-19. Lancet Neurol. 2020;19(9):767-783. doi:10.1016/S14744422(20)30221-0

20. Werneck AO, Vancampfort D, Oyeyemi AL, Stubbs B, Silva DR. Associations between TV viewing, sitting time, physical activity and insomnia among 100,839 Brazilian adolescents. Psychiatr Res. 2018;269:700-706. doi:10.1016/j.psychres.2018.08.101

21. Vancampfort D, Stubbs B, Firth J, Van Damme T, Koyanagi A. Sedentary behavior and depressive symptoms among 67,077 adolescents aged 12-15 years from 30 low- and middle-income countries. Int J Behav Nutr Phys. 2018;15:73. doi:10.1186/s12966-018-0708-y

22. Allen MS, Walter EE, Swann C. Sedentary behaviour and risk of anxiety: a systematic review and meta analysis. J Affect Disord. 2019. doi:10.1016/j.jad.2018.08.081

23. Fu W, Wang C, Zou L, et al. Psychological health, sleep quality, and coping styles to stress facing the COVID-19 in Wuhan, China. Transl Psychiatr. 2020;10(1). doi:10.1038/s41398-020-00913-3.

24. Callow DD, Arnold-Nedimala NA, Jordan LS, et al. The mental health benefits of physical activity in older adults survive the COVID-19 pandemic. Am J Geriatr Psychiatry. 2020;28 (10):1046-1057. doi:10.1016/j.jagp.2020.06.024

25. Jacob L, Tully MA, Barnett Y, et al. The relationship between physical activity and mental health in a sample of the UK public: a cross-sectional study during the implementation of COVID-19 social distancing measures. Ment Health Phys Act. 2020;19:100345. doi:10.1016/j.mhpa.2020.100345

26. Chen F, Zheng D, Liu J, et al. Depression and anxiety among adolescents during COVID-19: a cross-sectional study. Brain Behav Immun. 2020;88:36-38. doi:10.1016/j.bbi.2020.05.061

27. Zolotov Y, Reznik A, Bender S, Isralowitz R. COVID-19 fear, mental health, and substance use among Israeli university students. Int J Ment Health. 2020. doi:10.1007/s11469-020-00351-8

28. Satici B, Gocet-Tekin E, Deniz ME, Satici SA. Adaptation of the fear of COVID-19 scale: its association with psychological distress and life satisfaction in Turkey. Int J Ment Health. 2020. doi:10.1007/ s11469-020-00294-0

29. Dsouza DD, Quadros S, Hyderabadwala ZJ, Mamun MA. Aggregated COVID-19 suicide incidences in India: fear of COVID-19 infection is the prominent causative factor. Psychiatr Res. 2020;290:113145. doi:10.1016/j.psychres.2020.113145 
30. Chen S, Liu Y, Tremblay MS, et al. Meeting 24-hour movement guidelines: prevalence, correlates and the relationships with overweight and obesity among Chinese children and adolescents. J Sport Health Sci. 2020. doi:10.1016/j.jshs.2020.07.002

31. Cao R, Gao T, Hu Y, et al. Clustering of lifestyle factors and the relationship with depressive symptoms among adolescents in Northeastern China. $J$ Affect Disord. 2020;274:704-710. doi:10.1016/j.jad.2020.05.064

32. Hechi Government. Population of Hechi City. 2020

33. Wang J, Zou J, Luo J, et al. Mental health symptoms among rural adolescents with different parental migration experiences: a cross-sectional study in China. Psychiatr Res Neuroimaging. 2019;279:222-230. doi:10.1016/j.psychres.2019.03.004

34. Guang Y, Feng Z, Yang G, et al. Depressive symptoms and negative life events: what psycho-social factors protect or harm left-behind children in China? BMC Psychiatry. 2017;17(1). doi:10.1186/s12888-017-1554-1.

35. Statistics Bureau of Yizhou District, Hechi City. Statistical bulletin of national economic and social development in Yichou District of Hechi City in 2019. 2020

36. Zhou S, Zhang L, Wang L, et al. Prevalence and socio-demographic correlates of psychological health problems in Chinese adolescents during the outbreak of COVID-19. Eur Child Adolesc Psychiatry. 2020;29(6):749-758. doi:10.1007/s00787-020-01541-4

37. Schuch FB, Bulzing RA, Meyer J, et al. Associations of moderate to vigorous physical activity and sedentary behavior with depressive and anxiety symptoms in self-isolating people during the COVID-19 pandemic: a cross-sectional survey in Brazil. Psychiatry Res. 2020.

38. Faul F, Erdfelder E, Buchner A, Lang A. Statistical power analyses using $\mathrm{G}^{*}$ Power 3.1: tests for correlation and regression analyses. Behav Res Methods. 2009;41(4):1149-1160. doi:10.3758/ BRM.41.4.1149

39. Faul F, Erdfelder E, Lang AG, Buchner A. G*Power 3: a flexible statistical power analysis program for the social, behavioral, and biomedical sciences. Behav Res Methods. 2007;39(2):175-191. doi:10.3758/BF03193146

40. Craig CL, Marshall AL, Sj Str MM, et al. International physical activity questionnaire: 12-country reliability and validity. Med Sci Sport Exerc. 2003;35(8):1381-1395. doi:10.1249/01. MSS.0000078924.61453.FB

41. Macfarlane DJ, Lee CCY, Ho EYK, Chan KL, Chan DTS. Reliability and validity of the Chinese version of IPAQ (short, last 7 days). $J$ Sci Med Sport. 2007;10(1):45-51. doi:10.1016/j.jsams.2006.05.003

42. Liu X, Yang Y, Liu Z, et al. Psychometric properties of Youth Self-Rating Insomnia Scale (YSIS) in Chinese adolescents. Sleep Biol Rhythms. 2019;17(3):339-348. doi:10.1007/s41105-019-00222-3

43. Wang W, Bian Q, Zhao Y, et al. Reliability and validity of the Chinese version of the Patient Health Questionnaire (PHQ-9) in the general population. Gen Hosp Psychiatr. 2014;36(5):539-544. doi:10.1016/j.genhosppsych.2014.05.021

44. Leung DYP, Mak YW, Leung SF, Chiang VCL, Loke AY. Measurement invariances of the PHQ-9 across gender and age groups in Chinese adolescents. Asia-Pac Psychiatr. 2020. doi:10.1111/ appy. 12381

45. He X, Li C, Qian J, Cui H, Wu W. Reliability and validity of a generalized anxiety disorder scale in general hospital outpatients. Shanghai Arch Psychiatry. 2010;22(4):200-203.

46. Beard C, Björgvinsson T. Beyond generalized anxiety disorder: psychometric properties of the GAD-7 in a heterogeneous psychiatric sample. J Anxiety Disord. 2014;28(6):547-552. doi:10.1016/j. janxdis.2014.06.002

47. Chi X, Chen S, Chen Y, et al. Psychometric evaluation of the fear of COVID-19 scale among Chinese population. Int J Ment Health Addict. 2020.

48. Ahorsu DK, Lin C, Imani V, et al. The fear of COVID-19 scale: development and initial validation. Int J Ment Health. 2020. doi:10.1007/s11469-020-00270-8
49. Tang W, Wang G, Hu T, et al. Mental health and psychosocial problems among Chinese left-behind children: a cross-sectional comparative study. J Affect Disorders. 2018;241:133-141. doi:10.1016/j. jad.2018.08.017

50. Perogamvros L, Castelnovo A, Samson DR, Dangvu TT. Failure of fear extinction in insomnia: an evolutionary perspective. Sleep Med Rev. 2020;51:101277. doi:10.1016/j.smrv.2020.101277

51. Bendau A, Petzold MB, Pyrkosch L, et al. Associations between COVID-19 related media consumption and symptoms of anxiety, depression and COVID-19 related fear in the general population in Germany. Eur Arch Psy Clin. 2020.

52. Brown HE, Pearson N, Braithwaite RE, Brown WJ, Biddle SJH. Physical activity interventions and depression in children and adolescents. Sports Med. 2013;43(3):195-206. doi:10.1007/s40279012-0015-8

53. Liu M, Wu L, Ming Q, Wallander JL. How does physical activity intervention improve self-esteem and self-concept in children and adolescents? Evidence from a meta-analysis. PLoS One. 2015;10 (8):e0134804. doi:10.1371/journal.pone.0134804

54. Liu M, Wu L, Yao S. Dose-response association of screen time-based sedentary behaviour in children and adolescents and depression: a meta-analysis of observational studies. Brit J Sport Med. 2016;50 (20):1252-1258. doi:10.1136/bjsports-2015-095084

55. Kalak N, Gerber M, Kirov R, et al. Daily morning running for 3 weeks improved sleep and psychological functioning in healthy adolescents compared with controls. J Adolesc Health. 2012;51 (6):615-622. doi:10.1016/j.jadohealth.2012.02.020

56. Edwards MK, Loprinzi PD. Experimentally increasing sedentary behavior results in increased anxiety in an active young adult population. $J$ Affect Disord. 2016;204:166-173. doi:10.1016/j. jad.2016.06.045

57. Lubans D, Richards J, Hillman C, et al. Physical activity for cognitive and mental health in youth: a systematic review of mechanisms. Pediatrics (Evanston). 2016;138(3):e20161642-e20161642. doi:10.1542/ peds.2016-1642

58. Heijnen S, Hommel B, Kibele A, Colzato LS. Neuromodulation of aerobic exercise - a review. Front Psychol. 2016;6(6):1890. doi:10.3389/fpsyg.2015.01890

59. Budde H, Machado S, Ribeiro P, Wegner M. The cortisol response to exercise in young adults. Front Behav Neurosci. 2015;9:13. doi:10.3389/fnbeh.2015.00013

60. Lin T, Kuo Y. Exercise benefits brain function: the monoamine connection. Brain Sci. 2013;3(4):39-53. doi:10.3390/brainsci3010039

61. Belem Da Silva CT, de Abreu Costa M, Kapczinski F, et al. Inflammation and internalizing disorders in adolescents. Prog Neuropsychopharmacol Biol Psychiatry. 2017;77:133-137. doi:10.1016/j.pnpbp.2017.03.023

62. Werneck AO, Christofaro DGD, Ritti-Dias RM, et al. Does physical activity influence the association between depressive symptoms and low-grade inflammation in adults? A study of 8048 adults. Physiol Behav. 2020;223:112967. doi:10.1016/j.physbeh.2020.112967

63. Doré I, Sylvester B, Sabiston C, et al. Mechanisms underpinning the association between physical activity and mental health in adolescence: a 6-year study. Int J Behav Nutr Phys. 2020;17(1). doi:10.1186/s12966-020-0911-5.

64. Kandola A, Ashdown-Franks G, Hendrikse J, Sabiston CM, Stubbs B. Physical activity and depression: towards understanding the antidepressant mechanisms of physical activity. Neurosci Biobehav Rev. 2019;107:525-539. doi:10.1016/j.neubiorev.2019.09.040

65. Driver HS, Taylor SR. Exercise and sleep. Sleep Med Rev. 2000;4 (4):387-402. doi: $10.1053 /$ smrv.2000.0110

66. Halson SL. Sleep in elite athletes and nutritional interventions to enhance sleep. Sports Med. 2014;44(S1):13-23. doi:10.1007/s40279-014-0147-0

67. Kim T, Choi J, Lee H, Park Y. Associations between dietary pattern and depression in Korean adolescent girls. J Pediatr Adol Gynecol. 2015;28(6):533-537. doi:10.1016/j.jpag.2015.04.005 
68. Kohler CA, Freitas TH, Maes M, et al. Peripheral cytokine and chemokine alterations in depression: a meta-analysis of 82 studies. Acta Psychiatr Scand. 2017;135(5):373-387. doi:10.1111/acps.12698

69. Endrighi R, Steptoe A, Hamer M. The effect of experimentally induced sedentariness on mood and psychobiological responses to mental stress. Brit J Psychiatr. 2016;208(3):245-251. doi:10.1192/bjp.bp.114.150755

70. Stanczykiewicz B, Banik A, Knoll N, et al. Sedentary behaviors and anxiety among children, adolescents and adults: a systematic review and meta-analysis. BMC Public Health. 2019;19(1). doi:10.1186/ s12889-019-6715-3.

71. Fletcher EA, Salmon J, McNaughton SA, et al. Effects of breaking up sitting on adolescents' postprandial glucose after consuming meals varying in energy: a cross-over randomised trial. J Sci Med Sport. 2018;21(3):280-285. doi:10.1016/j.jsams.2017.06.002

72. Rollo S, Antsygina O, Tremblay MS. The whole day matters: understanding 24-hour movement guideline adherence and relationships with health indicators across the lifespan. J Sport Health Sci. 2020. doi:10.1016/j.jshs.2020.07.004

73. Schuch FB, Bulzing RA, Meyer J, et al. Associations of moderate to vigorous physical activity and sedentary behavior with depressive and anxiety symptoms in self-isolating people during the COVID-19 pandemic: a cross-sectional survey in Brazil. Psychiatr Res. 2020;292:113339. doi:10.1016/j.psychres.2020.113339
74. Biddle SJH, Asare M. Physical activity and mental health in children and adolescents: a review of reviews. Brit J Sport Med. 2011;45 (11):886-895. doi:10.1136/bjsports-2011-090185

75. Hoare E, Milton K, Foster C, Allender S. The associations between sedentary behaviour and mental health among adolescents: a systematic review. Int J Behav Nutr Phys. 2016;13(1):108. doi:10.1186/s12966-0160432-4

76. Trinh L, Wong B, Faulkner GE. The independent and interactive associations of screen time and physical activity on mental health, school connectedness and academic achievement among a population-based sample of youth. J Can Acad Child Adolesc Psychiatry. 2015;24(1):17-24.

77. Kavanaugh K, Moore JB, Hibbett LJ, Kaczynski AT. Correlates of subjectively and objectively measured physical activity in young adolescents. J Sport Health Sci. 2015;4(3):222-227. doi:10.1016/j. jshs.2014.03.015
Psychology Research and Behavior Management

\section{Publish your work in this journal}

Psychology Research and Behavior Management is an international, peer-reviewed, open access journal focusing on the science of psychology and its application in behavior management to develop improved outcomes in the clinical, educational, sports and business arenas. Specific topics covered in the journal include: Neuroscience, memory and decision making; Behavior modification and management; Clinical

\section{Dovepress}

applications; Business and sports performance management; Social and developmental studies; Animal studies. The manuscript management system is completely online and includes a very quick and fair peer-review system, which is all easy to use. Visit http://www. dovepress.com/testimonials.php to read real quotes from published authors. 